\title{
Development and use of a decision support tool for supporting the operation of Melbourne Water's drinking water reservoirs, Victoria, Australia
}

\author{
$\underline{\text { R. Mills }}{ }^{a}$, C. Dallimore ${ }^{a}$, C. O'Neill ${ }^{\mathrm{a}}$, K. Cinque ${ }^{\mathrm{b}}$, and S. Haydon ${ }^{\mathrm{b}}$ \\ ${ }^{a}$ HydroNumerics Pty Ltd, Leve 1, 272 Lygon St, Carlton, Victoria, Australia \\ b Melbourne Water Corporation, 100 Wellington Pde, East Melbourne, Victoria, Australia \\ Email: rianda.mills@hydronumerics.com.au
}

\begin{abstract}
Operators and managers of water resources have a responsibility to make reliable decisions in response to a broad range of drivers, from strategic planning requirements to unforeseen or unusual events. These decisions are made with varying levels of uncertainty. To increase their certainty in the management of its drinking water reservoirs Melbourne Water (MW) have implemented a real-time decision support tool. The Aquatic Real-time Management System (ARMS) utilises historical and real-time field data and numerical models for reporting, analysing and assessing reservoir conditions with improved certainty. The three-dimensional hydrodynamic model ELCOM is configured for six of MW's nine major drinking water reservoirs, and each reservoir model has undergone performance checks commensurate with the available field data. Field instruments record up-to-the-minute observations that are remotely acquired by ARMS and automatically applied to the models to execute real-time simulations and scenario forecasting.
\end{abstract}

The ability to run models in real-time allows the user to forecast a range of scenarios and compare possible outcomes, thereby reducing uncertainty in decisions relating to the operational management of reservoirs. The ARMS Graphic User Interface (GUI) was tailor-made to MW's specific needs. Time series of an observed or modelled variable may be plotted against reporting criteria and presented to the operator for quick assessment. Customisation of ARMS is a major advantage. All MW's discrete and continuous data sources may be imported, configured and analysed, streamlining use and interrogation of data. Custom plotting allows efficient communication of key information and model output to operators and managers. Data gaps are filled using basic algorithms there-by minimising downtime.

The on-going integration of field data, model output, reporting criteria, error analysis and gap identification in ARMS has been applied successfully at MW to investigate the fate and transport of post-fire debris flow in Upper Yarra Reservoir, and to conduct scenario analysis for complex operational decision-making, for example, assessing the dilution rate of desalinated water in Cardinia Reservoir.

The components of MW ARMS are continually improved and updated, such as improving model performance and customising GUI functions. In-house capability is built through training in the use of MW ARMS and interpretation of output.

Keywords: $\quad$ Decision support, uncertainty, water resources, hydrodynamic modelling 


\section{INTRODUCTION}

Operators and managers of water supplies make decisions about unforeseen and planned events, with varying levels of uncertainty in the decision-making process. Large water supply systems are complex, often comprising multiple ecosystems (for example lakes, rivers, bays) with competing demands from a range of stakeholders. Making decisions related to the management of these complex systems requires access to a range of information, from historical and real-time data to model output. Software systems which integrate databases, models and real-time information sources in a way decision-makers can use effectively are known as decision support systems (DSS) (Rizzoli \&Young 1997). The Aquatic Real time Management System (ARMS) is such a DSS. The Melbourne Water ARMS configuration assists with management of drinking water reservoirs by enabling simple interrogation of real-time and historical data, and scenario modelling of planned or unforeseen reservoir events, and their potential impacts on water quality. Real-time data interrogation and modelling provide the basis for taking action to mitigate adverse water quality impacts to ensure the best quality water is supplied to the consumer.

\section{ARMS OVERVIEW}

ARMS is a computer software program written in Java and may be run across a range of platforms, specifically supporting Mac OS X, Windows and Linux operating systems (Ewing et al., 2004). ARMS is an overarching 'virtual' database interfacing information from many sources, from real-time and historical datasets to model input and output, making it customisable for a given organisation or system. The simple ARMS GUI contains a drop-down menu of linked database resources, allowing easy access to all of the data required to manage a system. The top tier of the MW ARMS hierarchy contains the key reservoirs, and below each reservoir is listed the corresponding real-time Lake Diagnostic System (LDS) and SCADA resources, historical data resources and the three-dimensional hydro-dynamic model for that reservoir (Figure 1). Through ARMS, historical and real-time data may be viewed, plotted and interrogated and forecast scenarios can be configured, executed and analysed. Operational and scenario reports may be automatically generated and system alarms activated.

\section{FIELD DATA}

Operational management of MW's reservoirs requires access to key data including reservoir inflow and outflow, inflow temperature, and in lake dissolved oxygen (DO) concentration, turbidity and water temperature. These parameters are measured in the field using a variety of telemetered instruments, including flow meters and water quality sensors. Additionally, reservoir modelling requires data to 'force' the model boundary conditions and set the model initial conditions, comprised of the aforementioned datasets plus site-specific meteorological parameters including wind speed and direction, solar radiation, air

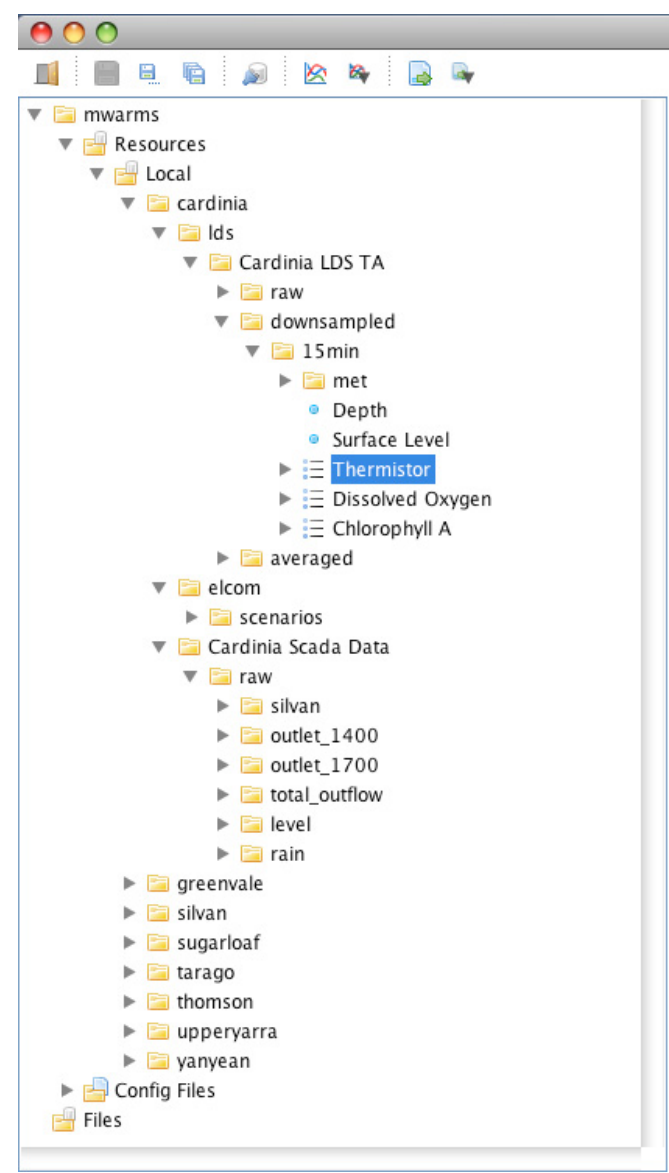

Figure 1. Drop-down menu in the MW ARMS GUI. temperature and relative humidity. MW have installed a network of 11 LDSs over 8 key reservoirs to provide time series of meteorological data, water temperature and DO concentrations over depth. LDS data are transferred in real-time to an onshore database 'LAKEMON' as simple text file files, while all other data are transferred to Melbourne Water's SCADA system. The SCADA system has been configured to export all required data streams to a text file at 6-minute intervals. These text files are then imported into the ARMS database. ARMS tasks are run to call and process these data for efficient access through the ARMS GUI. 


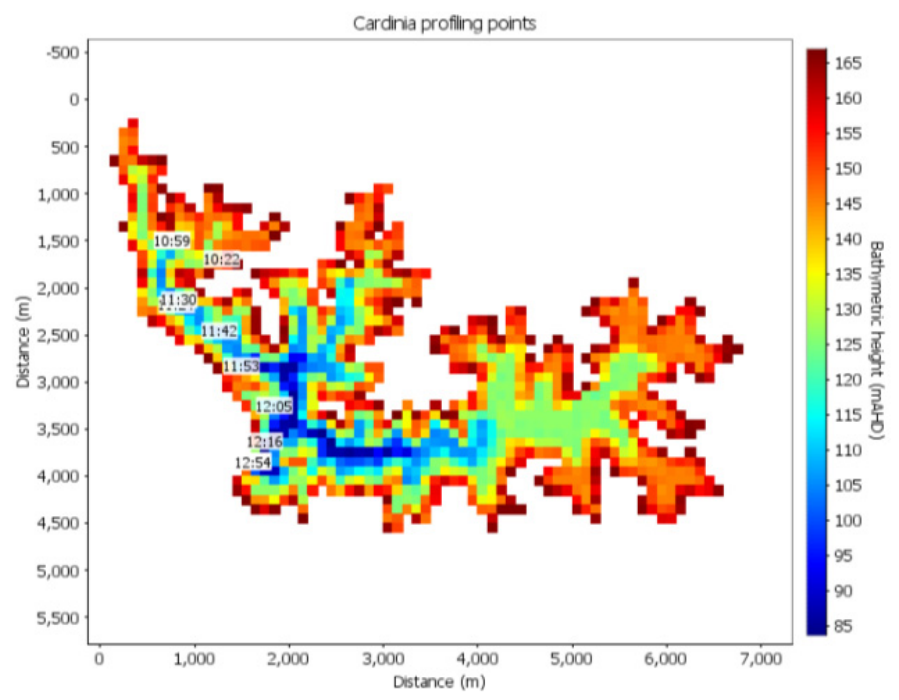

Figure 2. Example of profiling points plotted on a bathymetric map of Cardinia Reservoir. The figure was generated using GPS coordinates in MW ARMS. Numbers of the format HH:MM indicate the time the profile was taken. include quality checking and quality coding of raw data, down-sampling to time intervals suitable for model boundary condition files, filling of missing or bad quality data periods and averaging. To ensure users are aware when data filling has occurred filled data in time series plots are shown with vertical yellow lines (Figure 3).

A central function of ARMS is the ability to execute models (Ewing et al., 2004). Additionally, forcing data files must be of a certain file format, with specific units and date formats (refer to the ELCOM User Manual for more information (Hodges \& Dallimore, 2010)). The creation of forcing files is simplified through the ARMS GUI, which takes the user through the file creation process and contains a number of automated data processing algorithms. Forcing data may be manipulated for scenario modelling (for example, scaling the wind speed up or down) through a simple tool known as a wizard. The data processing method is recorded in the ARMS-generated forcing files.

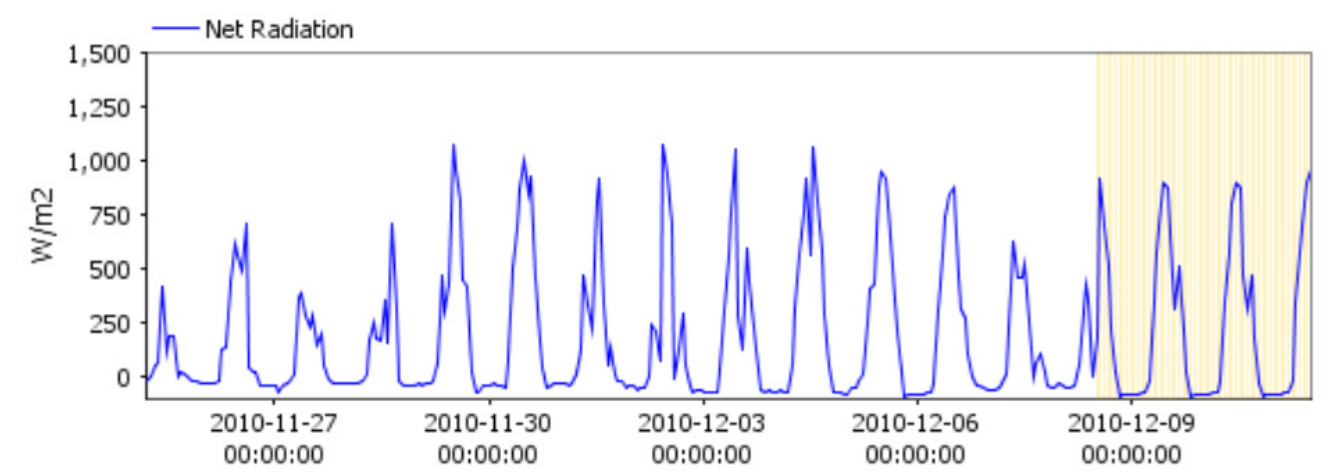

Figure 3. Net radiation data from the Cardinia Reservoir LDS. Vertical yellow lines from 2010-12-08 onward indicate where data gaps have been automatically infilled by ARMS.

\section{HYDRODYNAMIC AND WATER QUALITY MODELS}

Three-dimensional hydrodynamic models have been developed and validated to varying extents based on available data for Upper Yarra, Thomson, Silvan, Sugarloaf, Cardinia and Greenvale Reservoirs. These reservoirs are key drinking water supply reservoirs for Melbourne. The hydrodynamic model software used was the Estuary, Lake and Coastal Ocean Model (ELCOM) (Hodges \& Dallimore, 2011), which is a process based deterministic model for predicting temperature, salinity, density and velocity fields as driven by 
atmospheric and other boundary forcing. The Upper Yarra Reservoir and Greenvale Reservoir models will be explored here. These models were initially configured and validated by the Centre for Water Research.

\subsection{Upper Yarra Reservoir ELCOM}

Upper Yarra Reservoir (Figure 4) is located approximately $85 \mathrm{~km}$ east of Melbourne $\left(37.687^{\circ} \mathrm{S}, 145.917^{\circ} \mathrm{E}\right)$. The reservoir has a capacity of $200000 \mathrm{ML}$, a maximum height of main dam from river to crest of $90 \mathrm{~m}$ and a full supply surface area of 750 ha. Inflow is received from the surrounding, protected catchment (34 $000 \mathrm{ha})$ and transfers are supplied from Thomson Reservoir. The catchment of Thomson Reservoir is forested but unprotected with limited human access, meaning there is a small risk of human infectious pathogens being present in the reservoir and subsequently, being supplied to Upper Yarra Reservoir.

Upper Yarra Reservoir is an important part of Melbourne's drinking water supply; therefore sound management is required with respect to potential contamination from pathogens. ELCOM was developed and validated by way of a field experiment with the primary aim of determining the inflow dynamics and dilution characteristics of the controlled inflow from the Thomson Reservoir (Dallimore \& Imberger, 2006). The study consisted of three phases: (i) install monitoring equipment (2 LDS's), (ii) undertake numerical modelling and (iii)

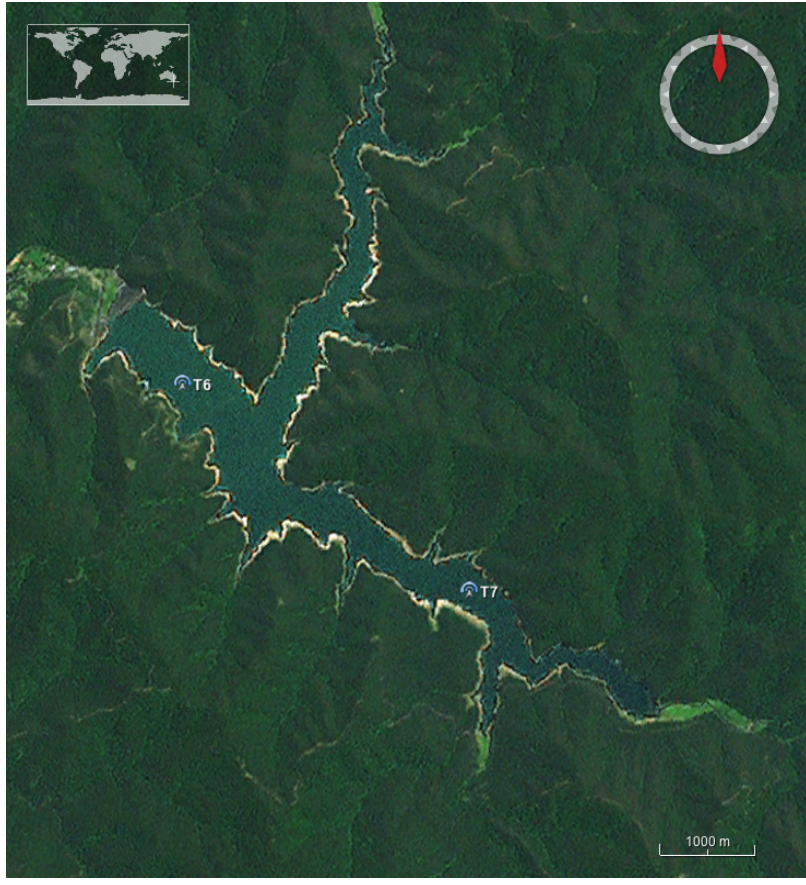

Figure 4. Upper Yarra Reservoir. The reservoir comprises two main 'arms'. The two LDS stations T6 and $\mathrm{T} 7$ are shown. complete a field experiment to investigate the Thomson inflow characteristics and dilution.

The geography of Upper Yarra Reservoir necessitated the installation of two sets of wind sensors to characterise the wind fields (important for reservoir mixing). As such, two LDS were installed in the reservoir (refer Figure 4). ELCOM was configured and the simulated water temperature compared well with the LDS data, with slight deviations attributed to large variations in wind fields across the reservoir surface. The model was also able to capture the depth, width and speed of the Thomson inflow intrusion when compared with data collected during the field experiment. Since its development, the Upper Yarra Reservoir ELCOM has been used to model several scenarios pertinent to reservoir management, including determining dilution of inflow from the Thomson Reservoir at the Upper Yarra offtake (Dallimore \& Imberger, 2006), design and modelling of a bubbler aerator for destratification (Dallimore, 2007) and the hydrodynamics of post-fire debris inflow into the reservoir (Mills \& Harris, 2011) (Mills, 2011). The latter of these is detailed later in section 5.2. 


\subsection{Greenvale}

Greenvale Reservoir (Figure 5) is located approximately $23 \mathrm{~km}$ north east of Melbourne (37.630o S, 144.902 o E). Greenvale has a capacity of 27000 ML, a maximum height of main dam from river to crest of $38 \mathrm{~m}$ and a full supply surface area of 174 ha. The reservoir supplies drinking water to north-western and western suburbs of Melbourne. It is classified as 'offstream' meaning the only inflows it receives are transferred from other reservoirs. Silica-based diatom growth in late winter and early spring can cause blockages in domestic water filters. To assess the factors affecting diatom growth, ELCOM was configured and coupled to the Computational Ecosystem DYnamics Model (CAEDYM) to model nutrients and key algal groups (Mills \& Harris, 2010). CAEDYM consists of a suite of sub-routines to calculate primary production, secondary production, nutrient and metal cycling and oxygen dynamics. The partially calibrated model has since been used to forecast the impact of transferring higher-nutrient water from Winneke Water Treatment Plant to Greenvale Reservoir.

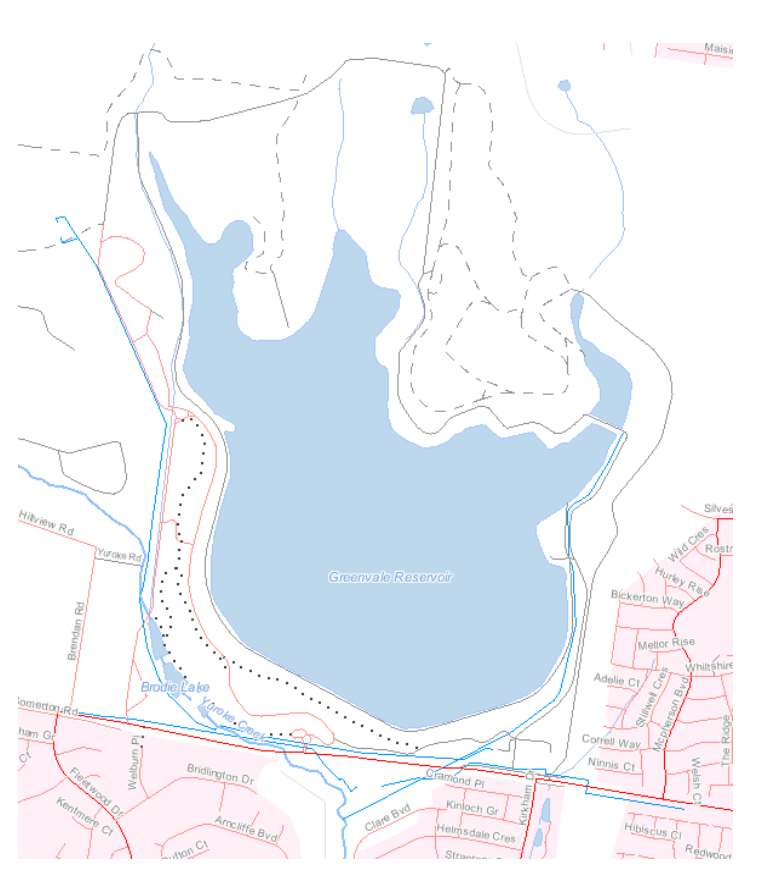

Figure 5. Greenvale Reservoir. Source: Victorian Water Resources

\section{ARMS CAPABILITIES}

MW use ARMS to view data pertinent to operational management and for numerical scenario modelling to inform incident management, strategic planning and research.

\subsection{Data interrogation}

Water temperature and DO field data are interrogated to inform operation of aerators during periods of strong stratification or when DO concentrations at depth begin to decline. MW ARMS contains several preconfigured plots, which automatically generate figures of LDS data including fortnightly DO depth profile time series, analysis of which can assist in efficient operation of aerators. In addition, the turbidity of influent water may be scrutinised to identify where further monitoring is required or when flow must be diverted or stopped due to elevated turbidity readings. Historical data is also at hand for identifying long-term trends.

\subsection{Scenario modelling}

MW ARMS contains a scenario 'wizard', a simple tool enabling quick set up of operational scenarios, such as altering a transfer volume or changing the source water. Modelling proposed operational changes allows for a quick assessment of the likely inflow dilution and/or hydrodynamics of the receiving reservoir, and an assessment of any likely water quality impacts. This section details two examples of how the reservoir models have been used in past scenario modelling exercises.

\subsubsection{Modelling the hydrodynamic response of post-fire debris inflow into Upper Yarra Reservoir}

A 3D hydrodynamic and water quality model of the Upper Yarra Reservoir was used to determine the reservoir's barrier capacity to a range of post-fire debris inflow scenarios. The study determined the times for modelled total suspended solids (TSS) to exceed then fall back below a particular criteria concentration near the reservoir offtake. Water may be selectively withdrawn from Upper Yarra Reservoir at different depths. Analysis of model output included investigation of the range of response times for shutting off water transfers, and the effect this had on TSS concentrations at different depths.

The results varied greatly dependent upon the time of year at which the debris flow event occurred, the location where the inflow entered and the magnitude of antecedent rainfall events. It took between 3 days and 3 months for TSS to reach a level of $5 \mathrm{mg} / \mathrm{L}$ at the offtake (the criteria concentration), and between 0.4 days and 11 months for TSS to fall below this criteria concentration (Mills, 2011). Figure 6 depicts TSS time 
series for 5 of the scenarios illustrating the variability in TSS at the offtake over time and for different inflow locations, loads and temperatures.

The Melbourne Water ARMS was upgraded to enable easy plotting and visualisation of key model outputs by reservoir operators and managers. Melbourne University determined influent ash loads, sediment loads and inflow volumes as required by the model.

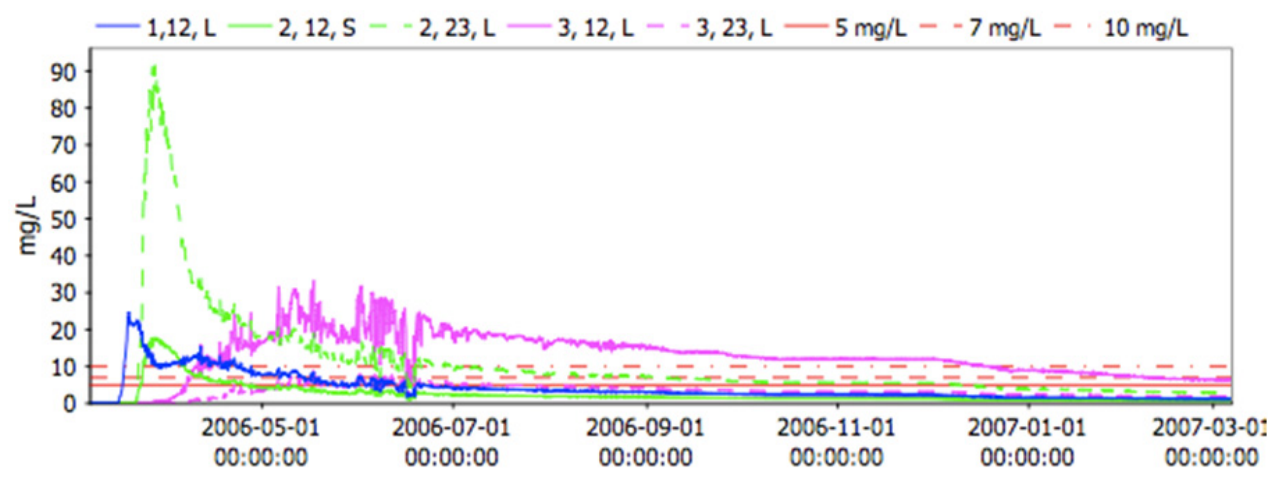

Figure 6. TSS concentrations near the offtake. Early autumn post-fire debris flow scenarios. 5, 7 and $10 \mathrm{mg} / \mathrm{L}$ criteria lines are in red. Source: Mills, 2011.

\subsubsection{Cardinia Reservoir desalination water inflow study}

The Cardinia Reservoir ELCOM model was configured, validated and utilised to determine the best location for the Wonthaggi desalination inlet structure to maximize dilution. Scenarios were run for each season, and for three different desalination inlet locations, 'east', 'saddle', and 'tower' Figure 7). It was recommended not to locate the desalinated inflow at the tower as scenario modelling showed little mixing between ambient and influent water. Locating the inflow at the eastern location resulted in greatest overall dilution of influent water prior to extraction at the offtake tower (Yeates et al., 2007). However, the 'Saddle' location provided adequate mixing and was significantly cheaper to construct therefore was ultimately chosen as the inlet location.

\section{CUSTOMISATION, CONTINUAL IMPROVEMENT AND TRAINING}

The fully customisable nature of ARMS ensures that it is readily updated to import new data sources and models, and generate custom plots and reports of pertinent data or model results. Data pre-processing may also be improved by applying more involved, statistically based gap filling. Melbourne Water may continually improve the model performance by further validating/calibrating and updating the models when new data becomes available. HydroNumerics are training Melbourne Water management and operational personnel to use ARMS.

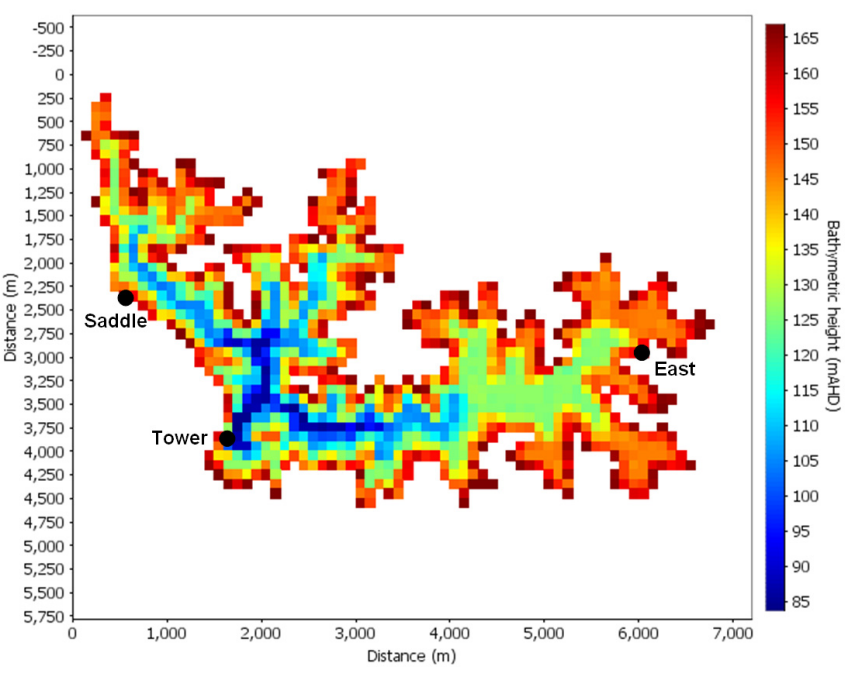

Figure 7. Cardinia Reservoir ELCOM bathymetry showing the three possible locations for desalination inflow: 'Saddle', 'Tower' and 'East'. The colour bar is bathymetric height in $\mathbf{m ~ A H D , ~ w i t h ~ d a r k ~ b l u e ~}$ indicating deep regions and dark red indicating shallow regions. After Yeates et al., 2007. 


\section{CONCLUSIONS}

Operators and mangers of water resources are required to make decisions in response to a broad range of operational or strategic drivers. Melbourne Water ARMS is an integrated DSS used to improve confidence in decision-making regarding management of their drinking water resources. It is essentially a wrap-around and single access point for real time and historical data and numerical models pertinent to making operational and strategic reservoir management decisions. Relevant data are transferred into ARMS, pre-processed to fill gaps and custom figures easily generated to view information of importance. A simple scenario wizard is used to create operational scenarios regarding water transfers. ARMS is customisable therefore is continually developed as management needs evolve, more field monitoring instruments become available or models are improved. Melbourne Water's Decision Support System MW ARMS is a key instrument in reducing uncertainty in the management of its drinking water resources.

\section{REFERENCES}

Dallimore, C.J. (2007). Hydrodynamic Simulation of the Upper Yarra Aerator Design. Centre for Water Research report.

Dallimore, C. J. and Imberger, J. (2006). Upper Yarra Reservoir Thomson Inflow Study. Centre for Water Research report.

Ewing, T., Romero, J.R., Imberger, J., Antenucci, J. and Deen, A. (2004). A real-time reservoir decision support system. Paper presented at the 6 th International Conference on Hydroinformatics, $21^{\text {st }}$ to $24^{\text {th }}$ June 2004, Singapore. ISBN 981-238-787-0.

Hodges, B. and Dallimore, C (2011). Estuary, Lake and Coastal Ocean Model: ELCOM v2.2 Science Manual. Centre for Water Research, University of Western Australia.

Hodges, B. and Dallimore, C (2010). Estuary, Lake and Coastal Ocean Model: ELCOM v2.2 User Manual. Centre for Water Research, University of Western Australia.

Mills, R. and Harris, J. (2010). Greenvale Reservoir Water Quality Modelling: Preliminary Data Analysis and Modelling. Centre for Water Research draft report.

Mills, R. (2011). Post-fire debris flow into Upper Yarra Reservoir: Hydrodynamic Modelling of Suspended Solids Fate and Transport (Phase 2). HydroNumerics Pty Ltd draft report HN MW 002.

Mills, R. and Harris, J. (2011). Post Fire Debris Flow into Upper Yarra Reservoir: Hydrodynamic Modelling of Suspended Solids Fate and Transport. Centre for Water Research report.

Rizzoli, A.E. and Young, W.J. (1997). Delivering environmental decision support systems: software tools and techniques. Environmental Modelling \& Software. Vol. 12, (2-3), pp 237-2491.

Yeates, P.S., Hillmer, I. and Antenucci, J.P. (2007). Cardinia Reservoir Desalination Water Inflow Study. Centre for Water Research report. 